\section{Panorámica de la cultura ambiental en la Universidad Autónoma de Zacatecas. México 2006-2016}

\section{Resumen}

La Universidad Autónoma de Zacatecas, desde su fundación y hasta el momento actual ha estado comprometida con la sociedad y el medio ambiente. En el año de 2006 presentó su proyecto de Cultura Ambiental ante CECADESU-SEMARNAT y ANUIES, este proyecto es transversal a todas las actividades sustantivas y adjetivas de la institución, acorde con su modelo académico UAZ siglo XXI.

Del año 2006-2012 se trabajó en Gestión Ambiental: Manejo y cuidado del agua y energía, procesos de forestación, espacios 100\% libres de humo de tabaco, manejo de residuos no peligrosos y peligrosos, no uso de unicel. De 2009-2016 se inició el trabajo de Ambientalización de las Curriculas, acompañando estos procesos con formación en Cultura Ambiental de alumnos, profesores y trabajadores a través de conferencias, talleres, un diplomado y retroalimentando en investigación la realización de 4 simposios internacionales y 5 congresos internacionales. La UAZ forma parte de la RED de planes institucionales de Cultura Ambiental. Organizados por CECADESU-SEMARNAT-ANUIES. Palabras clave: Cultura-Ambiental, Universidad Autónoma de Zacatecas.
María Alejandra Moreno García', Claudia Maldonado Tapia', Elda Araceli García Mayorga ${ }^{3}$, Jesús Rivas Gutiérrez², Luis Enrique Crespo Jiménez ${ }^{4}$ José Jesús Muñoz Escobedo 2

1 Investigador Docente, Unidad Académica de Ciencias Biológicas.

2 Investigador Docente, Instituto de Investigaciones Odontológicas/UAO.

3 Investigador Docente, Unidad Académica de Enfermería.

4 Alumno de la Maestría de Ciencias Biológicas. UAZ. Universidad Autónoma de Zacatecas. México.

Cuerpo Académico de Biología Celular y Microbiología UAZ-103. Cuerpo Académico de Diabetes y Enfermedades Relacionadas UAZ165.

Correspondencia:

झ”amoreno_29@hotmail.com

\title{
Summary
}

The Autonomous University of Zacatecas (UAZ), from its founding to the present has been committed to society and the environment. In 2006 the university presented the project Environmental Culture at CECADESU-SEMARNAT and ANUIES, which is transverse to all substantive and adjectival activities of the institution, according to its academic model UAZ XXI century. 
From year 2006 to 2012 the University has worked in Environmental Management: Management and care of water and energy, forestation processes, $100 \%$ smoke-free spaces, management of non-hazardous and hazardous waste, no use of Styrofoam. From 2009-2016 Greening the Curricula work began, accompanying these processes with Environmental Culture training of students, teachers and workers through lectures, workshops, and feeding back a diploma in conducting research, 4 international symposia and conferences. UAZ is part of the institutional network plans of Environmental culture .Organized by CECADESU-SEMARNAT-ANUIES.

Key words: Environmental Culture, Universidad Autónoma de Zacatecas.

\section{Introducción}

Nuestra Alma Mater es una institución que en el transcurso de sus ciento ochenta y cuatro años ha desempeñado, en cada momento de su historia, un papel central en la Educación de los Zacatecanos, pues vive y toma conciencia sobre la importancia del desarrollo sociocultural, económico, y la importancia de nuestro momento actual ante el cambio climático y las consecuencia de este en todos los ámbitos de nuestras vidas a consecuencia del aumento de la población mundial, procesos de desforestación masiva, pérdida de la biodiversidad de plantas y animales, contaminación del agua, aire, suelo, impactando esto en nuestra calidad de vida y salud, de ahí la importancia que las nuevas generaciones a su egreso de las instituciones de educación lleven una visión ambiental de respeto a la naturaleza no solo su conservación si no hacia un desarrollo sostenible, que favorezca una mejor calidad de vida de todos los habitantes del planeta Tierra.

Con fecha del 13 de septiembre de 1774, el H. Ayuntamiento de esta capital de Zacatecas México se dirigió al Virrey de la Nueva España, solicitando la creación de un Colegio. El Padre Jesuita Don Francisco Pérez de Aragón, eminente zacatecano, donó a este Colegio las ganancias de la Hacienda de Linares, correspondiente a Fresnillo, la que había generado al Conde de Regla $\$ 85,676.00$ pesos y le regaló también una casa sobre cuyo terreno se levantó el actual edificio del ayuntamiento.

Al ocupar la gubernatura del Estado el señor Francisco García Salinas "Tata Pachito", como le llamaba el pueblo fue quien promovió en 1832 la fundación de una Casa de Estudios.

Esta primera Casa de Estudios que funcionó en la Ciudad de Jerez García Salinas desde el 5 de noviembre de 1832 (por decreto) hasta el 27 de abril de 1837 y luego se trasladó al edificio de la ciudad de Zacatecas (antiguo Colegio de San Luis Gonzaga).

Ya en Zacatecas desde su primer Director, el Lic. Teodosio Lares y los que lo sucedieron, le dieron un gran impulso al nuevo plantel, los planes de estudio se reformaron y fueron cada vez más operantes.

En 1870 se creó la Escuela de Enfermería, que con el paso del tiempo resulto ser una de las más 

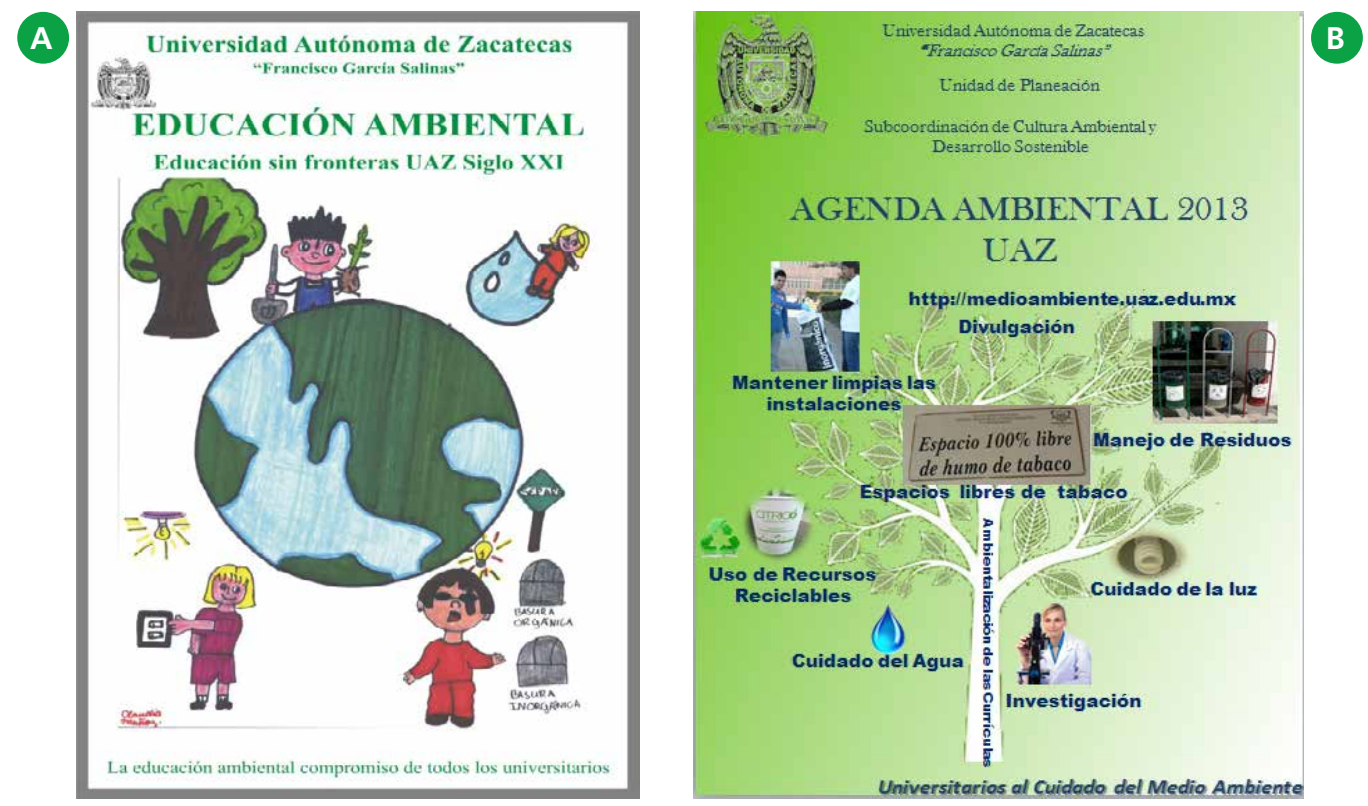

Figura 1. Agenda Ambiental UAZ. (A) Utilizada en 2006-2012, (B) 2013-2016.

prestigiadas del país. En ese año también se inició los estudios de Ingeniería.

A partir de 1885 el Instituto Científico y Literario de García se transformaría en "Instituto Científico y Literario de Zacatecas".

Las tendencias nacionalistas emanadas de la Revolución Mexicana, la influencia del Estado en nuevas esferas de la vida social cambiarían el rumbo del Instituto que en sólo dos años cambia de nombre. En 1918 fue denominado "Colegio del Estado" y a partir de 1920 se le denominó "Instituto de Ciencias de Zacatecas".

En 1958 se enfrenta la lucha por la autonomía que es conquista por los estudiantes zacatecanos y se otorga el reconocimiento por parte del Estado el 10 de octubre de 1959, denominándose "Instituto de Ciencias Autónomo de Zacatecas" (ICAZ). El mismo estaba integrado por las siguientes escuelas: Secundaria, Preparatoria, Enfermería, Ingeniería y Derecho.

Durante el período gubernamental del ingeniero José Isabel Rodríguez Elías, se permitió que el Instituto de Ciencias Autónomo de Zacatecas, mediante decreto del 6 de septiembre de 1968, el ejecutivo del Estado lo transformara en la Universidad Autónoma de Zacatecas (1).
En 1999 inició un proceso de reforma, culminado con la nueva Ley Orgánica, avalada por la Cámara de diputados del Estado de Zacatecas, con el objetivo de elevar la calidad académica y la pertinencia social y la puesta en marcha del nuevo modelo académico UAZ siglo XXI.

Dando origen a una nueva estructura académica conformada por áreas del conocimiento, las cuales están constituidas por Unidades y Programas.

En 2005 se inicia el trabajo con el nuevo modelo académico UAZ siglo XXI el cual está centrado en el estudiante y el compromiso de formar estudiantes integrales no solo en el campo disciplinar también axiológico y profesional y se inauguran nuevas instalaciones que albergan la primera Área Ciencias de la Salud conformada por las Unidades de Ciencias Químicas (Programa de Químico-Fármaco-Biólogo), Unidad Académica de Odontología (Programa de Lic en Médico Cirujano Dentista), Unidad Académica de Medicina Humana y Ciencias de la Salud (Programa de Médico Cirujano), Unidad Académica de Enfermería (Programa de Lic. en Enfermería) y el programa de Nutrición (Lic. en Nutrición), que posteriormente pasa a ser parte de la Unidad Académica de Enfermería. 

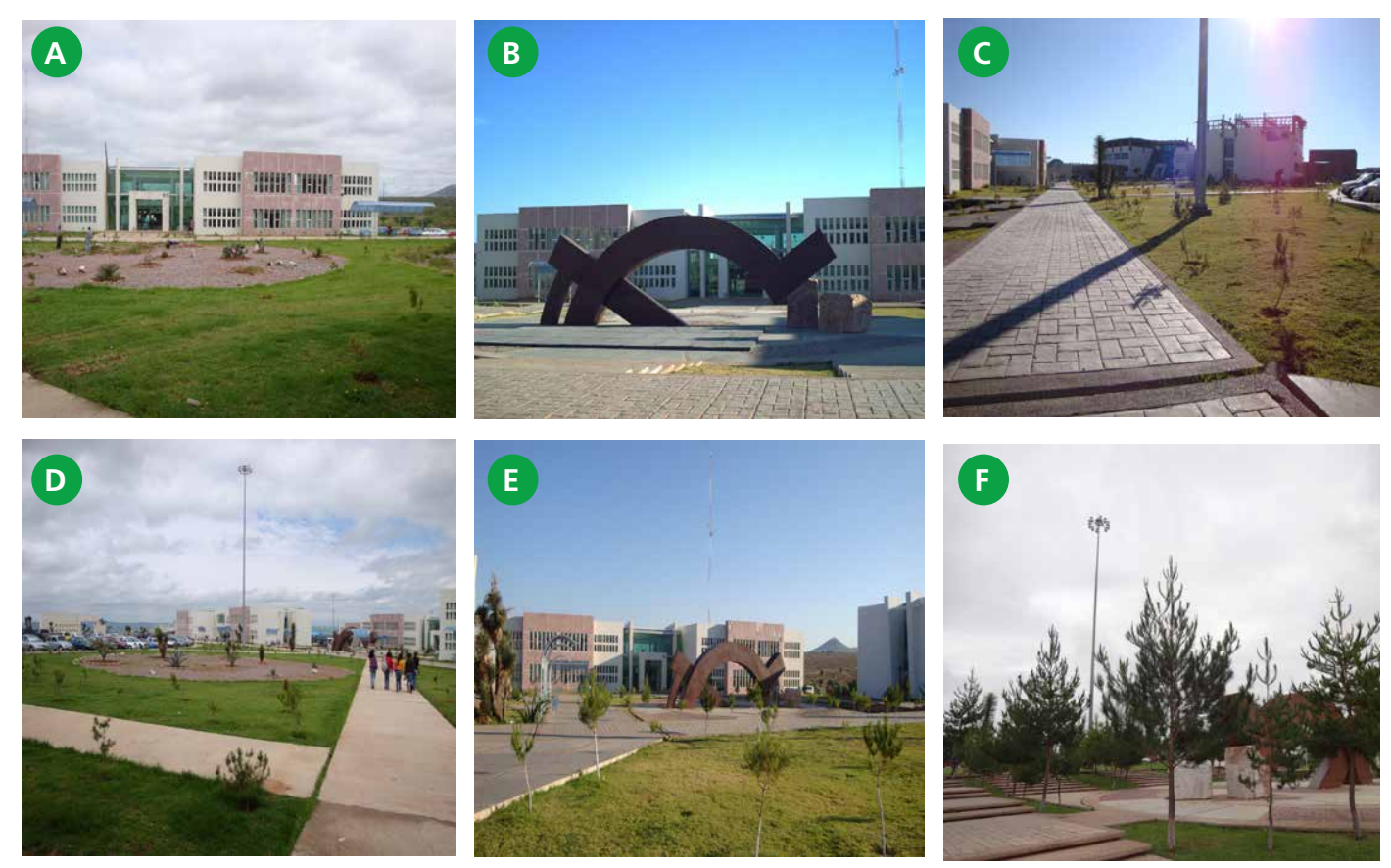

Figura 2. Evolución del desarrollo del proceso de forestación en el Campus UAZ Siglo XXI (2008-2016).

La Universidad Autónoma de Zacatecas siempre ha manifestado su intencionalidad en lo que se conoce como misión institucional, la cual consiste en educar y formar profesionistas emprendedores, responsables y honestos. Sustentada además, en una sólida formación humanística, científica, tecnológica y respetuosa del medio ambiente.

En el nuevo modelo académico de nuestra Universidad, se establece tambien un compromiso con la educación ambiental acorde con el plan nacional de educación ambiental 2005-2015 (2, 3, 4).

Actualmente la población Universitaria está conformada por 40,116 estudiantes, distribuidos en: 40 licenciaturas, 11 especialidades, 30 maestrías y 8 doctorados además de los 13 planteles de preparatoria y uno de secundaria, que conforman la amplia oferta educativa de la Máxima Casa de Estudios, en sus modalidades escolarizada, semiescolarizada y en línea, en 30 Unidades. Está presente actualmente en 19 municipios del estado y es la máxima instancia académica del estado, en 2008 ingresó al Consorcio de Universidades Mexicanas (CUMex) que aglutina a las 29 Universidades de excelencia de México.

\section{La Cultura Ambiental para la sostenibilidad en la UAZ}

El nuevo proyecto ambiental para la Universidad, es un planeamiento participativo, incluyente, autónomo, plural, abierto, flexible, comprensivo e integral.

Es un documento en construcción con ajustes, actualizaciones y en constante movimiento de adecuación, para favorecer la construcción de una cultura ambiental en busca de una mejor calidad de vida de sus integrantes y de la sociedad y el cuidado del medio ambiente.

\section{UAZ siglo XXI}

Las Universidades como instituciones de educación y en donde su función primordial es formar recursos de calidad, actualmente también tienen la responsabilidad de impulsar la cultura para la sostenibilidad no solo en el marco de sus estudiantes, docentes y trabajadores sino en la sociedad en general.

Con la cual están comprometidos, todos en un trabajo colegiado compartiendo la responsabilidad y el compromiso de entregar a las generaciones futuras un espacio educativo, un estado y un país con 


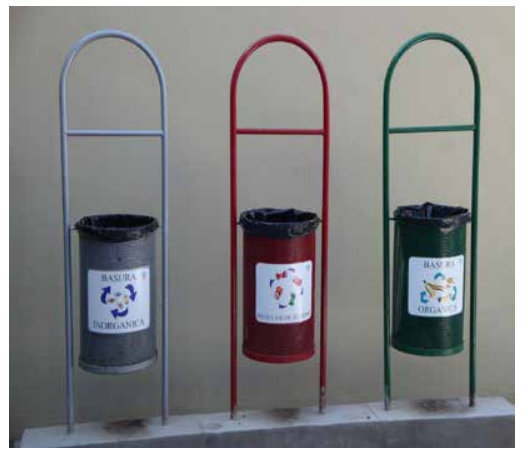

Figura 3. Contenedores instalados en todos los espacios universitarios para separación de residuos: aluminio, residuos inorgánicos y orgánicos.

un medio ambiente que les permita aspirar a una vida de calidad y digna.

La Estrategia de Cultura Ambiental para la sostenibilidad en la UAZ es la puesta en marcha y el compromiso de los universitarios por sentar las bases con su compromiso con el medio ambiente y la sostenibilidad. Así mismo está enmarcada por los compromisos internacionales asumidos por México.

En el año 2005 la ONU decretó que hasta el año 2010 sería, el decenio de la educación ambiental y la socialización de la información. En el año 2006 la UAZ presentó a ANUIES-CECADESU-SEMARNAT el proyecto Estrategias de Educación Ambiental UAZ siglo XXI $(2,3,4,5)$, recibiendo apoyo para su cum- plimiento en el 2007, por PRODERIC, instancia de apoyo a la investigación del Gobierno de Zacatecas.

De 2007 a 2015 se ha presentado a ANUIESCECADESU-SEMARNAT el seguimiento del proyecto en la UAZ.

\section{Objetivo general}

Consolidar la Cultura Ambiental para la sostenibilidad como una política institucional fundamentada en los lineamientos legislativos de la Ley Orgánica de la Universidad Autónoma de Zacatecas y transversal a la legislación del estado y del país, que tenga como fin la construcción de una Cultura Ambiental en la Universidad Autónoma de Zacatecas que impacte en la sociedad de Zacatecas, favoreciendo mejorar las condiciones de la madre tierra hacia la sostenibilidad.

\section{Material y métodos}

1. Socialización de la estrategia, establecimiento de una agenda ambiental: Gestión Ambiental y Ambientalización de las Curriculas.
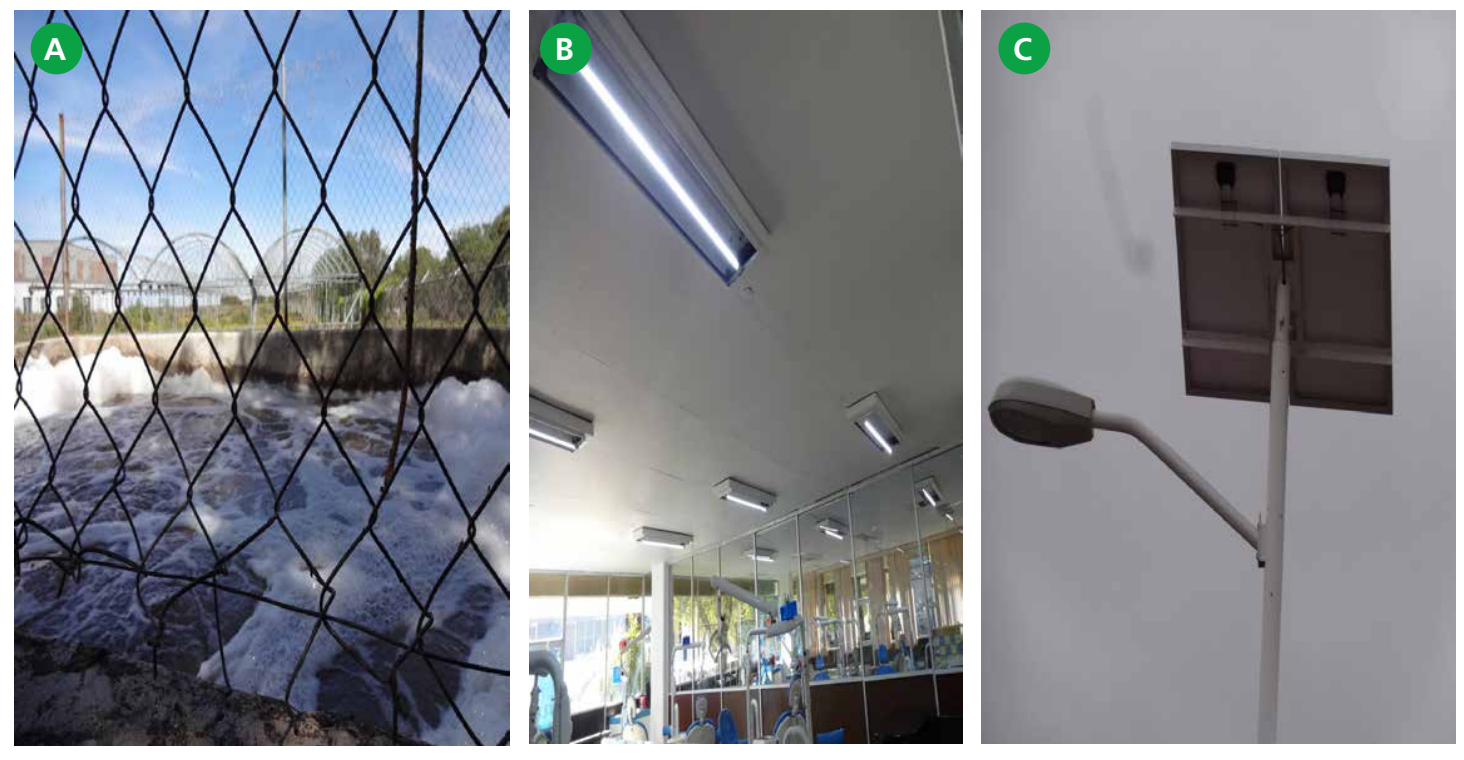

Figura 4. (A) Planta tratadora de aguas primera fase. (B) Utilización de lámparas ahorradoras en una clínica odontológica de la UAZ. (C) Lámpara con celdas solares. 

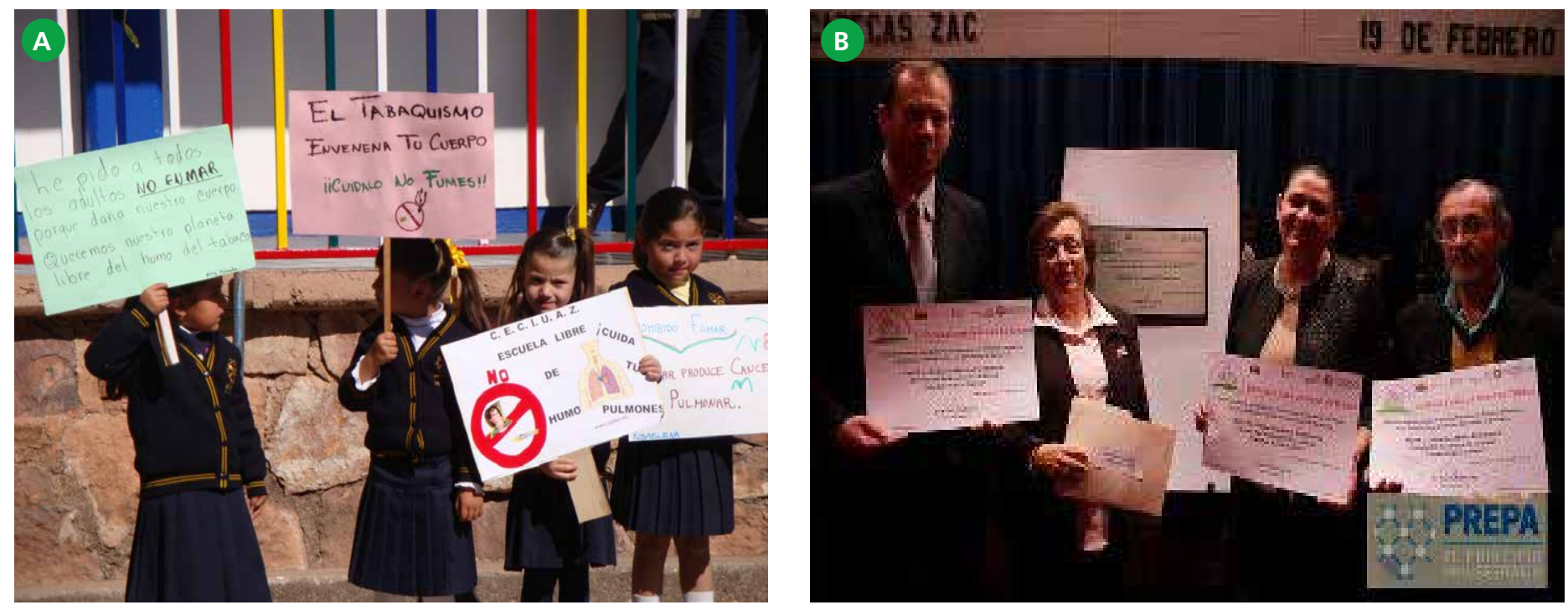

Figura 5. (A) Alumnos de guardería de la Universidad Autónoma de Zacatecas el día de su proceso de certificación como espacio 100\% libre de humo de tabaco y (B) directores de unidades académicas de la UAZ el día de su certificación como espacios 100\% libres de humo de tabaco.

2. Interacción de la educación formal (Realizar foros para fomentar el conocimiento sobre cambio climático y desarrollo sostenible, catalogo de investigadores de la UAZ que trabajen proyectos a favor del medio ambiente) y no formal en educación ambiental trasversal para la formación integral de nuestros estudiantes, docentes y trabajadores.

3. Aplicar un adecuado manejo de los Residuos orgánicos, inorgánicos, biológico infecciosos y peligrosos generados en las diversas Unidades Académicas de la UAZ.

4. Efectuar acciones de forestación y reforestación dentro de los Campus Universitario UAZ.

5.No uso de Unicel.

6. Reciclaje de aluminio.

7. Recolección de pilas.

8. Generar espacios $100 \%$ libres de Humo de Tabaco.

9. Implementar el programa de administrativo sostenible (PAS).

10. Biosustentabilidad de los integrantes de la comunidad Universitaria.

11. Optimización en el uso y manejo del agua y la energía.

12. Ambientalización de las Curriculas.
13. Dx visual 2012-2013 del estado de Cultura Ambiental en la UAZ.

Las presentes acciones se llevaron a cabo por el Cuerpo Académico de Biología Celular y Microbiología con la cooperación de la comunidad Universitaria y con el apoyo de la autoridades Universitarias, solo con la articulación de todo se ha podido avanzar en este trabajo. Así mismo ha recibido apoyo de PRODERIC/Zacatecas 2007, PIFI-SEP 2010-20112012-2013, Red-PRODEP-SEP 2015.

\section{Resultados}

1. El Proyecto se difundió en espacios académicos de la UAZ, se colocó en todos los espacios la agenda ambiental.

2. Se participó en la organización de 5 congresos internacionales de Cambio Climático y Desarrollo Sostenible 2009-2016 (dos organizados por la Universidad Autónoma de Zacatecas, uno por la Universidad Autónoma de Nuevo León, y dos por La universidad Nacional de la Plata en Argentina y como producto académico: se publicaron dos libros de memorias digitales con ISBN, dos libros 


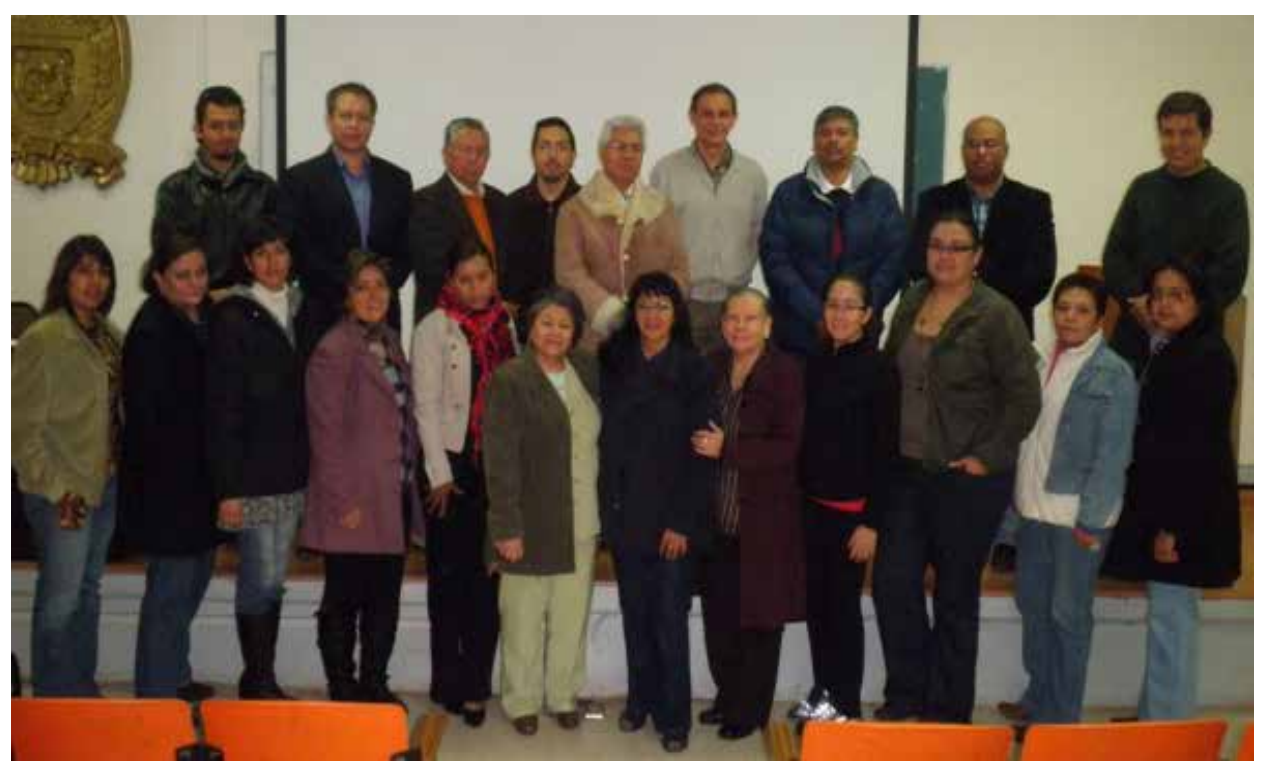

Figura 6. Primer grupo de docente que llevó a cabo el taller de Educación Ambiental impartido por la Maestra María Teresa Bravo Mercado. UNAM. 2012

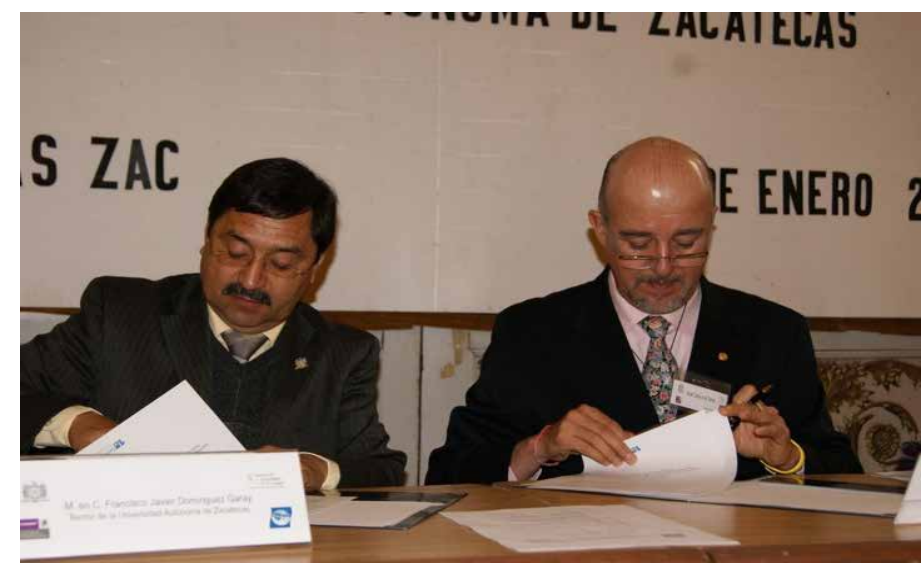

Figura 7. Firma de compromiso de la Carta de la Tierra, por parte de la UAZ el Rector Francisco Javier Domínguez Garay y por el representante de México por parte de la sociedad civil, ante el foro social de América Latina y el Caribe del programa de las Naciones Unidas para el medio ambiente (2012).

impresos con ISBN, dos números especiales en la Revista Latinoamericana de Bioquímica Clínica con ISSN, con contenidos temáticos cuidado del agua, energía, manejo de residuos, biodiversidad, recursos naturales, desarrollo urbano, etc.)

3. Se colocaron contenedores para separación de residuos orgánicos, inorgánicos y aluminio en los diferentes espacios de la UAZ.

4. Se llevó a cabo 5 procesos de forestación.

5. Se tiene catalogo de investigadores en medio ambiente en la UAZ.
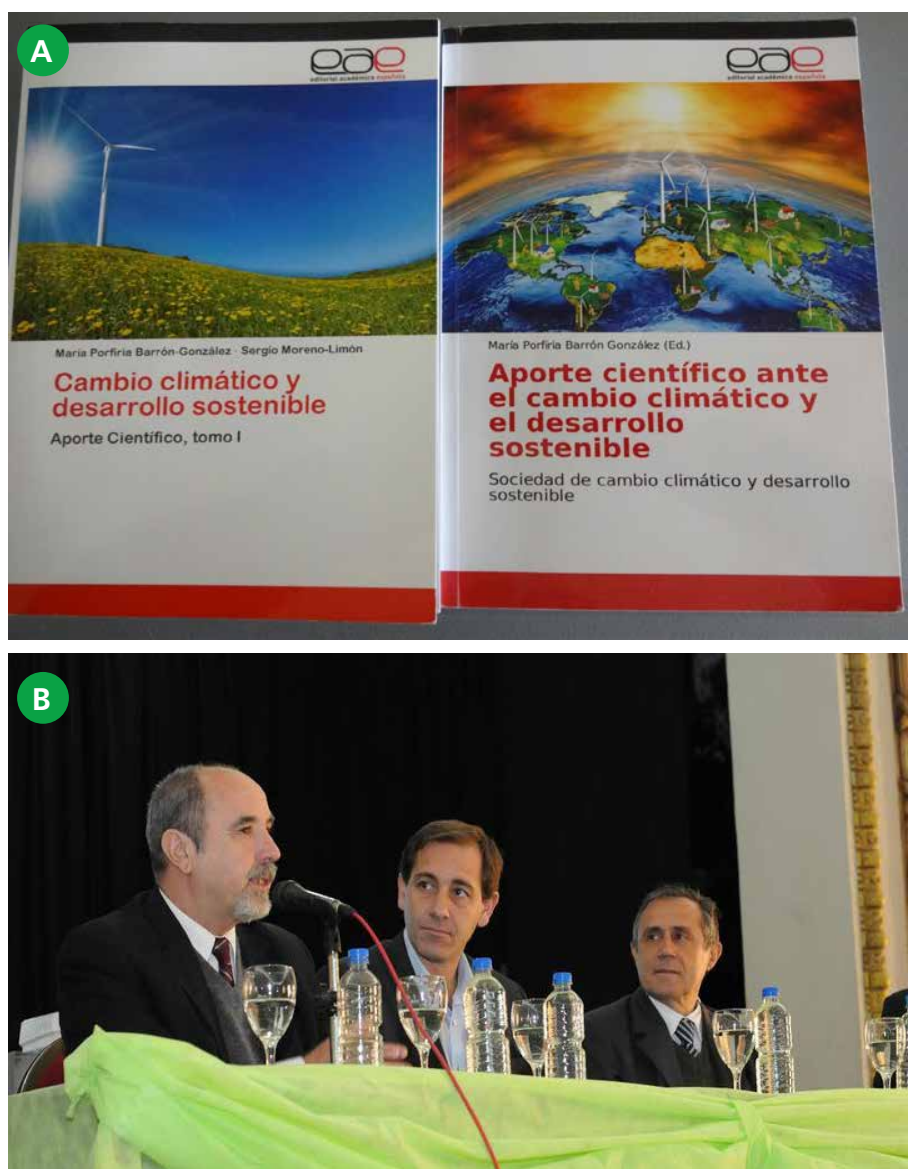

Figura 8. (A) Se muestra Figura de los libros publicados de los trabajos en extenso (2016) del cuarto congreso internacional de Cambio Climático y Desarrollo Sostenible en 2013. (B) Inauguración del quinto congreso internacional de Cambio Climático y Desarrollo Sostenible por el Presidente de la Universidad Nacional de la Plata el Dr. Raúl Perdomo en la Ciudad de la Plata Argentina, septiembre 2016. 
6. 50 espacios de la UAZ fueron declarados $100 \%$ libres de humo de tabaco.

7. Se publicó el primer manual de manejo de residuos peligrosos y biológico infecciosos del Áreas de Ciencias de la Salud, de Básicas y Agropecuarias con ISBN.

8. Se adquirió el compromiso con la Carta de la Tierra.

9. Dentro de la UAZ se han desarrollado 3 simposios de Cultura Ambiental y Desarrollo Sostenible y como producto dos números especiales de la revista de Investigación Científica de la UAZ con ISSN, 10.-Se cuenta con el primer diagnostico sobre la Ambientalización Curricular en la UAZ. 11.-Realización del Dx visual de Cultura Ambiental en la UAZ 2012-2013.

\section{Seguimiento del proceso de Cultura}

Ambiental: resultados del auto diagnostico. Diagnostico visual de Octubre-2012-Febrero 2013

Referente a las áreas verdes en el Campus UAZ siglo $X X I$, se observa un desarrollo favorable del $75 \%$, en el año 2012, en Zacatecas se presentó una sequia intensa y desafortunadamente se perdieron arboles de más de metro y medio de altura.

En la Guardería infantil, y Medicina Veterinaria un $90 \%$ fue favorable, y en Humanidades un $20 \%$ por falta del recurso hídrico.
En la UAZ existe un problema del manejo de sus residuos, no se colocan adecuadamente en los contenedores para su separación, no hay disminución de volumen, reciclado, reutilización y no existe espacios para el almacenamiento de estos, los residuos que generan los universitarios son de tipo inorgánico: plastico, vidrio, aluminio, papel, unicel, plástico y en menor proporción orgánicos, Faltan espacios universitarios en tener su manual de manejo de Residuos Peligrosos.

Existen espacios universitarios que de manera habitual hay falta de agua, la luz continua encendida en horas de luz solar.

El gasto de luz anual de 2012 (información generada por la secretaría de Finanzas de la UAZ). \$2, 489,564.00, pesos mexicanos (gráfica 1).

Gasto de Agua anual 2012 (Información generada por la secretaría de Finanzas de la UAZ), no está contemplado el gasto en construcción. \$1, $827,187.00$ redes de agua. $\$ 166,576.00$ pipas, pesos mexicanos (gráfica 2).

Existen ceniceros para las colillas de cigarros y se continúan depositando en el piso, el parque vehicular no está en condiciones idóneas, la utilización del unicel es habitual, existe contaminación visual, auditiva, del aire.

Es muy importante señalar en este diagnostico, que en muchos espacios universitarios, está

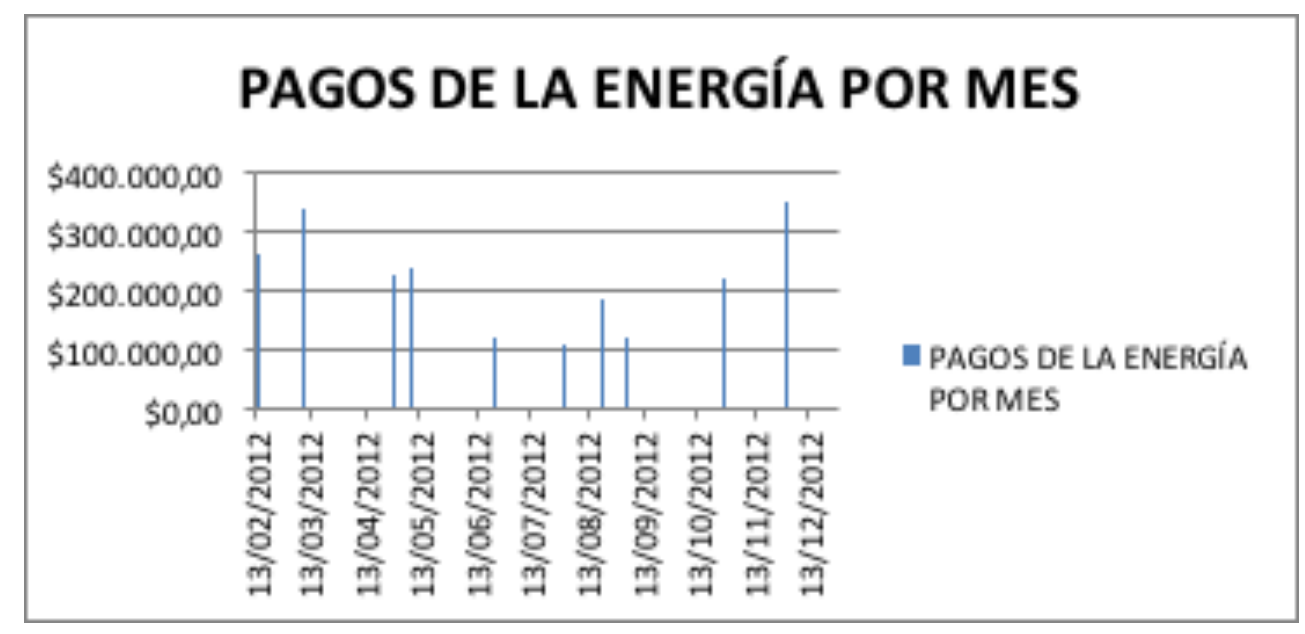

Gráfica 1. El costo de energía en la UAZ en 2012, el total. $\$ 2,489,564.00, \mathrm{M} / \mathrm{N}$. 


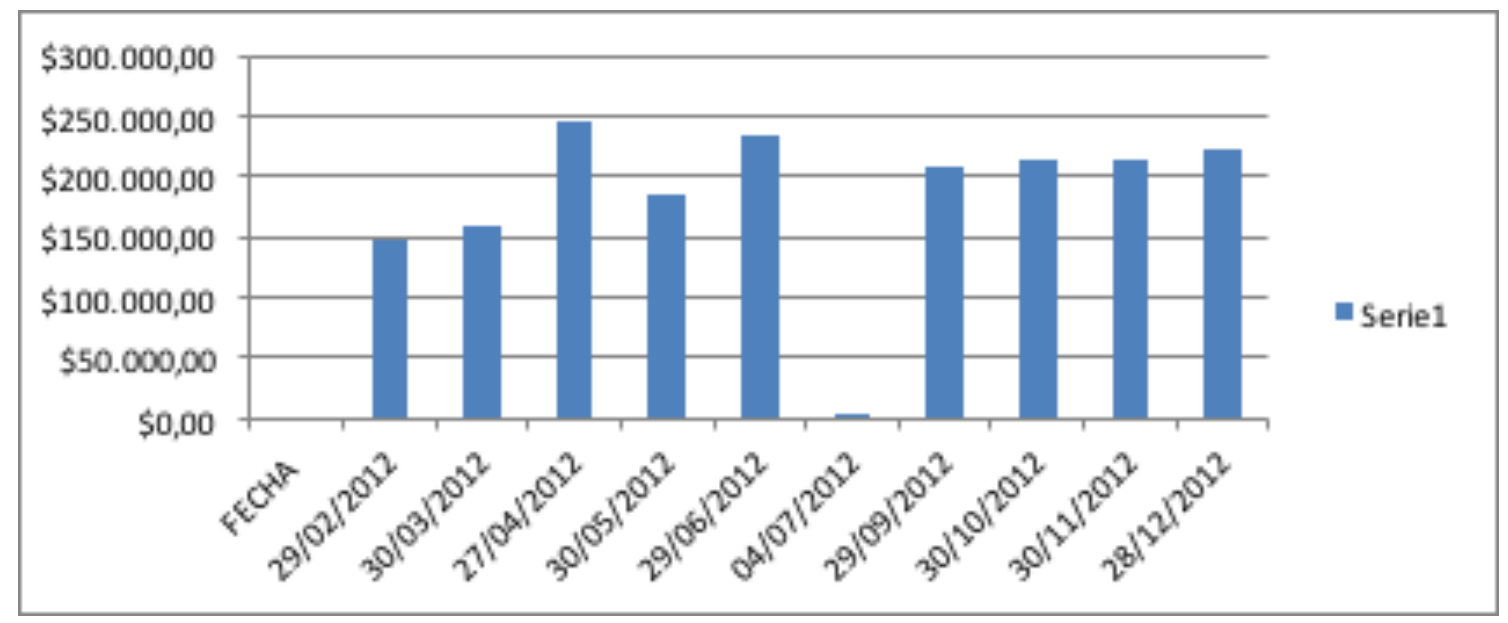

Gráfica 2. El costo de agua durante el año 2012. \$1, 827,187.00 M/N.

permeando la Cultura Ambiental, sus espacios se mantienen limpios, está la señalética, y hay presencia de imagen verde (Figura 9).

El presente trabajo recibió apoyo de PRODERICZacatecas 2007 y de PIFI-SEP 2011, 2012 y 2013, Red-PRODEC-SEP 2015.

\section{Discusión}

Educar para el desarrollo sostenible significa incorporar los temas fundamentales del desarrollo sostenible a la enseñanza y el aprendizaje, por ejemplo: el cambio climático, la reducción del riesgo de
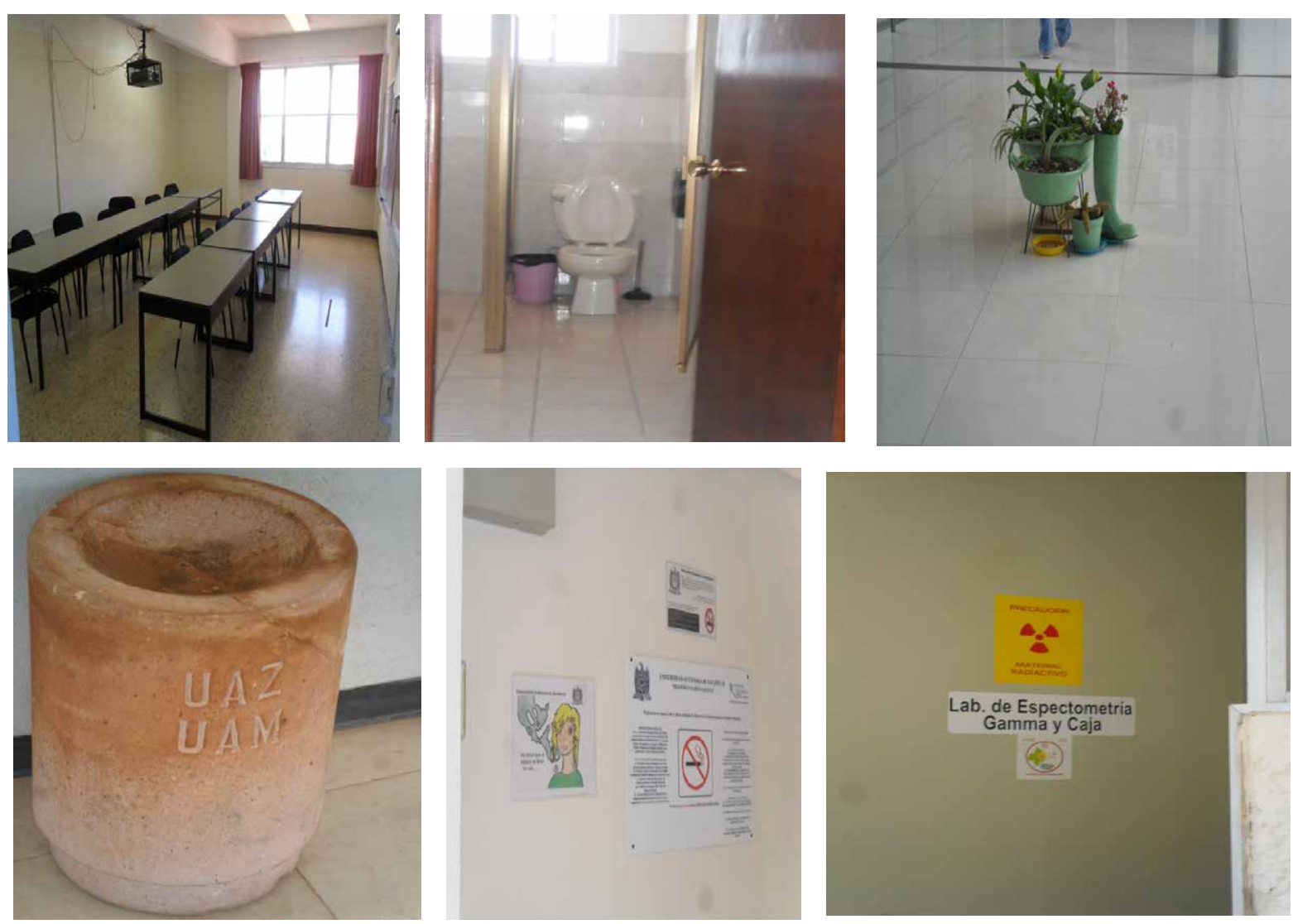

Figura 9. Se muestra una composición de imágenes de espacios limpios, señalética y vegetación. 
desastres, la biodiversidad, la reducción de la pobreza y el consumo sostenible. Asimismo, las Instituciones de Educación Superior exigen métodos participativos de enseñanza y aprendizaje que motiven a los estudiantes y les doten de autonomía, a fin de cambiar su conducta y facilitar la adopción de medidas en pro del desarrollo sostenible $(10,11)$. De ahí la necesidad de que la comunidad universitaria refuerce su compromiso con la naturaleza, somos la institución líder del conocimiento en Zacatecas y tenemos la responsabilidad de formar estudiantes con una formación integral que cuiden su entorno y de dejar condiciones adecuadas a las generaciones futuras.

El proyecto de Cultura Ambiental de la Universidad Autónoma de Zacatecas tiene 10 años de iniciado su primera fase se enfocó a la realización de acciones de Gestión Ambiental, implementando infraestructura, pero esto no es suficiente, de ahí la necesidad de complementar con la Ambientalización de las Curriculas.

Hay mucho trabajo por realizar, se implementaron acciones y trabajo colegiado pero el camino por recorrer aun es largo, y el compromiso es doble no solo como comunidad tener un desarrollo sostenible, tenemos la obligación de fortalecer la cultura ambiental en nuestra sociedad y mitigar los efectos del cambio climático que nos ha impactado en todas las actividades de nuestra vida.

La comunidad Universitaria es una de las poblaciones más importantes del estado de Zacatecas México, en donde confluyen de todos los municipios, de todos los sectores de la sociedad. Por ende estamos relacionados a la vez con todos los sectores del estado, en donde se privilegia la docencia, investigación, extensión, vinculación y difusión de la cultura y nuestro objetivo es formar estudiantes integrales comprometidos con su sociedad, el momento crítico que vive la humanidad por el cambio climático, con todas las modificaciones del medio ambiente las grandes sequias, las abundantes inundaciones, los ciclones, los terremotos, los procesos de deforestaciones, los incendios forestales, la per- dida de la biodiversidad y el aumento de la población mundial en todos sus requerimientos para vivir, sobre todo el alimentario, en salud y educación, el aumento de problemas de salud por vectores como es el Dengue, Zika, Chinkuguya, activación de enfermedades reemergentes como la tuberculosis, problemas digestivo por contaminación del agua y alimentos, el uso y abuso de medicamentos, y el consumo de alimentos elaborados con agroquímicos, hormonas, etc.

Sí las Universidades no toman su papel de responsabilidad, como agentes de cambio el futuro no es promisorio, por eso estamos seguros que donde esté un alumno, un profesor hay acciones que realizar a favor de todos, trabajemos de manera conjunta y todo será mejor, el que un niño tenga una alimentación sana, salud y educación es tarea de todos y solo trabajando con responsabilidad, trasparencia y equidad tendremos un mejor mundo y regresar un poco de todo lo que nos ha dado la

\section{Madre Tierra.}

El diagnóstico en la UAZ, es un área de oportunidad para el cuidado de la naturaleza.

Vivimos en una era ambientalista nos guste o no.

Todas las fuerzas que acaban con el planeta son las fuerzas del hombre. No permitamos que esto ocurra, unamos esfuerzos y voluntades, para rediseñar la historia encaminada hacia la conservación y lograr el desarrollo sostenible.

El manejo adecuado y separación de los residuos orgánicos, inorgánicos y aluminio, al reducir su volumen, reciclar y reutilizar, son una fuente de recursos económicos.

El optimizar el uso del agua y energía, permitirá disminuir los costos y que el vital líquido no falte en los diferentes espacios universitarios.

En un inicio se tienen que invertir recursos económicos, pero los beneficios no son solo en este sentido, sino también en lo social y mejorar nuestras condiciones ambientales, fortaleciendo la cultura del respeto al medio ambiente. 
Las Instituciones de Educación Superior, son el espacio de la formación de recursos humanos en diferentes campos disciplinares, y esta formación tiene que tener impacto a través de toda su vida, debe ser una formación integral en su disciplina, lo cultural, lo deportivo, en las nuevas tecnologías, pero con una responsabilidad en lo ambiental y un desarrollo sostenible.

Las acciones de Gestión Ambiental son fundamentales, pero deben tener la retroalimentación de la Ambientalización Curricular.

Estas acciones favorecen mejorar la salud de todos, incluyendo a la Madre Tierra.

\section{Conclusión}

En la Comunidad de la UAZ se ha socializado la estrategia y adquirió el compromiso con el medio ambiente de realizar acciones tanto en la educación formal, como no formal del cuidado del medio ambiente y por ende de la Madre Tierra.

\section{Bibliografía}

1. Hurtado Bonilla. JL. www.uaz.edu.mx/ Consultado el 2 de septiembre de 2014.

2. SEMARNAT. www.semarnat.gob.mx/ Consultado el 20 de marzo de 2014.

3. CONAGUA. www.cna.gob.mx/ Consultado el 30 de abril de 2014.

4. CECADESU. www.semarnat.gob.mx/educacionambiental. Consultado el 27 de agosto de 2015.

5. ONU. www.un.org/es/ Consultado el 30 de junio de 2015.

6. UNESCO. www.unesco.org/new/es/unesco/ Consultado el 7 de julio de 2013.

7. PROFEPA. www.profepa.gob.mx/ Consultado el 6 de junio de 2014.
8. REMEXMAR. www.remexmar.uaz.edu.mx Consultado el 2 de septiembre de 2015.

9. DECLARACIÓN DEL MILENIO. www.un.org/es/ Consultado el 30 de octubre de 2015.

10. Belausteguigoitia Rius Juan Carlos. Avances en la institucionalización del desarrollo sustentable en México. Reflexiones y avances hacia un desarrollo sustentable en México. Obtenido el día 26 de mayo 2007 de, http://www.redmeso.net

11. Delgadillo Escalona. El desarrollo sustentable en México 19802007. 2007. Revista Digital Universitaria. Vol. 9. No. 3. Pág. 1-13.

Material fotográfico: Claudia Yadira Muñoz Moreno y Yersinia Alejandra Muñoz Moreno.

\section{Opina sobre este artículo:}
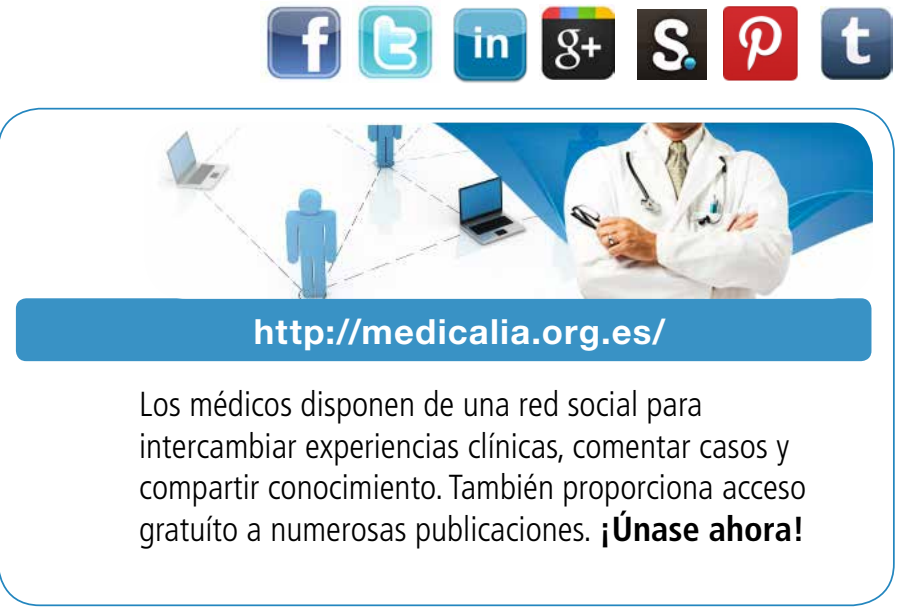

\section{Publique con iMedPub}

\section{http://www.imed.pub}

\section{Biomedicina}

$\checkmark$ Biomedicina es una revista de acceso abierto en Español, dedicada a todas las áreas de la medicina, tanto básicas como clínicas.

$\checkmark$ Recoge artículos de investigación originales, revisiones y casos clínicos. 